\title{
The critical influence of some "tiny" geometrical details on the stress field in a Brazilian Disc with a central notch of finite width and length
}

\author{
Stavros K. Kourkoulis, Christos F. Markides, Ermioni D. Pasiou \\ National Technical University of Athens, School of Applied Mathematical and Physical Sciences, Department of Mechanics, \\ Laboratory for Testing and Materials, Theocaris Building, Zografou Campus, 157 73, Athens, Greece \\ stakkoun@central.ntua.gr, bttp:/ /orcid.org/0000-0003-3246-9308 \\ markidib@mail.ntua.gr,http://orcid.org/0000-0001-6547-3616 \\ epasion@teemail.gr, http://orcid.org/0000-0003-1580-3415 \\ Andronikos Loukidis, Dimos Triantis \\ University of West Attica, Department of Electrical \& Electronics Engineering, Electronic Devices and Materials Laboratory, \\ 250 Thivon Avenue, 122 44, Athens, Greece \\ a.loukidis@uniwa.gr, http://orcid.org/0000-0002-5764-5366 \\ triantis@uniwa.gr,bttp://orcid.org/0000-0003-4219-8687
}

\begin{abstract}
The role of some geometrical characteristics of the notches machined in circular discs, in order to determine the mode-I fracture toughness of brittle materials, is discussed. The study is implemented both analytically and numerically. For the analytic study advantage is taken of a recently introduced solution for the stress- and displacement-fields developed in a finite disc with a central notch of finite width and length and rounded corners. The variation of the stresses along strategic loci and the deformation of the perimeter of the notch obtained analytically are used for the calibration/validation of a flexible numerical model, which is then used for a parametric investigation of the role of geometrical features of the notched disc (thickness of the disc, length and width of the notch, radius of the rounded corners of the notch). It is concluded that the role of the width of the notch is of critical importance. Both the analytic and the numerical studies indicate definitely that ignoring the accurate geometric shape of the notch leads to erroneous results concerning the actual stress field around the crown of the notch. Therefore, it is possible that misleading values of the fracture toughness of the material of the disc may be obtained.
\end{abstract}

KEYwORDs. Brazilian disc; Central notch; Analytical solution; Numerical analysis; Finite elements.

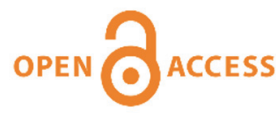

Citation: Kourkoulis, S.K., Markides, Ch.F., Pasiou, E.D., Loukidis, A., Triantis, D., The critical influence of some "tiny" geometrical details on the stress field in a Brazilian Disc with a central notch of finite width and length, Frattura ed Integrità Strutturale, 59 (2022) 405422.

Received: 11.11 .2021

Accepted: 21.11 .2021

Published: 01.01.2022

Copyright: (C) 2022 This is an open access article under the terms of the CC-BY 4.0, which permits unrestricted use, distribution, and reproduction in any medium, provided the original author and source are credited. 


\section{INTRODUCTION}

$\mathrm{M}$ ode-I fracture toughness, $\mathrm{K}_{\mathrm{IC}}$, is a critical mechanical parameter for structural engineering applications because it quantifies the resistance of materials against the initiation (and propagation) of pre-existing cracks due to tensile loads acting normally to the crack axis. Both ASTM and ISRM have long ago standardized the procedure for the laboratory determination of $\mathrm{K}_{\mathrm{IC}}[1,2]$. Especially for brittle, rock-like materials, three standardized tests are usually adopted: The "Short Rod", the "Chevron Bend" and the "Cracked Chevron Notched Brazilian Disc (CCNBD)" ones. The latter is, perhaps, the one most widely used worldwide. However, and in spite of its wide application, some issues concerning the validity of its outcomes are still under study [3,4] and some critical questions are still open [5]. Among them special attention is paid to: (i) the reasonability of the formulae available for the determination of the respective Stress Intensity Factor (SIF), (ii) the exact shape of the cracks machined and (iii) the actual boundary conditions prevailing along the disc-jaw contact arc.

Currently, the formulae adopted for the determination of the SIF in the CCNBD test are obtained from the respective "Cracked Straight Through Brazilian Disc" (CSTBD) configuration, i.e., considering a disc with a central "mathematical" crack (i.e., a linear discontinuity with zero distance between its lips and singular tips) loaded by a pair of diametral forces. It is obvious, however, that in practical applications the cracks machined are rectangular notches rather than mathematical cracks [6] and the disc is loaded by a complicated combination of radial (and shear) stresses rather than by a pair of point forces $[7,8]$. Things become even more complicated taking into account that for obvious practical restrictions the "cracks" machined cannot be "short" enough (with respect to the radius of the disc) and, therefore, the common assumption that the boundary conditions do not influence the stress field in the immediate vicinity of the crack becomes questionable.

In this direction, an attempt is described in this study to address some of the aforementioned issues. The main target is to quantify the influence of some "tiny" geometrical details on the stress field (and, therefore on the respective SIF or, equivalently, on the value of $\mathrm{K}_{\mathrm{IC}}$ ) in a Brazilian Disc with a central notch of finite width and length and rounded corners. In addition, in the analytic approach, the loading scheme comprises a parabolic distribution of radial stresses, acting along two finite arcs. The specific loading scheme is proven to approach extremely closely the stress field developed during the compression of the disc between the curved jaws suggested by ISRM [2] (Fig.1a). The geometrical details considered in this study include: (i) the length of the notch with respect to the radius of the disc, (ii) the width of the notch, (iii) the radius of the corners of the notch, and, finally, (iv) the thickness of the disc.

As a first step the problem is confronted analytically by means of the complex-potentials technique [9]. Analytical (but lengthy) full-field formulae, in series form, are obtained for the components of the stress- and displacement-fields. Taking into account the difficulties to handle these expressions for practical purposes and exhaustive parametric investigations, a numerical model is developed, calibrated and validated with the aid of the analytic solution. The numerical model is then used for the parametric study of the role of the geometrical characteristics (length, width and corner-radius) of the notch. Moreover, the numerical model is used to enlighten the influence of the third dimension (i.e., that along the disc's thickness), a parameter that is not taken into account by the analytic solution, which is based on two-dimensional elasticity.

The results of the study indicate clearly that ignoring the exact geometric shape of the notch may lead to erroneous results concerning the stress field in the immediate vicinity of the crowns of the notch and therefore to unacceptable results concerning the actual fracture toughness of the disc's material.
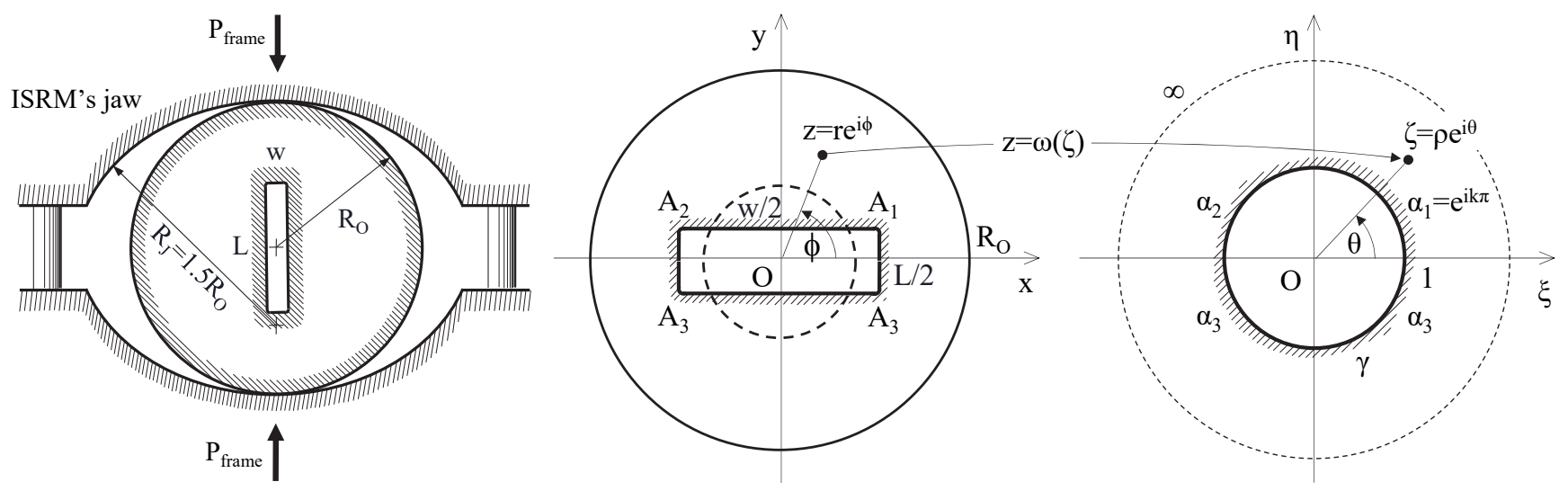

(a)

(b)

Figure 1: (a) Schematic representation of the notched disc squeezed between the curved jaws suggested by ISRM [2]; (b) The mapping function provided by Savin [11], which was used for the analytic solution of the problem. 


\section{ANALYTICAL SOLUTION}

$\mathrm{F}$

rom the theoretical point of view, the problem of the finite circular disc with a rectangular notch (of finite length and width) with rounded corners, squeezed between the curved jaws suggested by the respective ISRM standard (Fig.1a), is here confronted as a first fundamental problem of the plane theory of linear elasticity. In this context, and assuming, in addition, that the material of the disc is homogeneous and behaves isotropically, Muskhelishvili's complex potentials technique is adopted [9]. The general solution of the problem was obtained, relatively recently, by Markides and Kourkoulis [4,10] by taking advantage of the following mapping function, provided by Savin [11] (Fig.1b):

$$
\begin{aligned}
& z=\omega(\zeta)=R\left(\zeta+\sum_{\ell=1}^{\infty} \frac{c_{\ell}}{\zeta^{\ell-1}}\right), \quad R=\frac{b}{2}\left[1+\sum_{\ell=1}^{\infty}(-1)^{\ell} c_{\ell}\right]^{-1} \\
& c_{1}=\frac{\beta+\bar{\beta}}{2} ; \quad c_{2}=\frac{(\beta-\bar{\beta})^{2}}{24} ; \quad c_{3}=\frac{\left(\beta^{2}-\overline{\beta^{2}}\right)(\beta-\bar{\beta})}{80} ; \quad c_{4}=\frac{5\left(\beta^{4}+\overline{\beta^{4}}\right)-4\left(\beta^{2}+\overline{\beta^{2}}\right)-2}{896} ; \\
& c_{5}=\frac{7\left(\beta^{5}+\overline{\beta^{5}}\right)-5\left(\beta^{3}+\overline{\beta^{3}}\right)-2(\beta+\bar{\beta})}{2304} ; \quad c_{6}=\frac{21\left(\beta^{6}+\overline{\beta^{6}}\right)-14\left(\beta^{4}+\overline{\beta^{4}}\right)-5\left(\beta^{2}+\overline{\beta^{2}}\right)-4}{11264} \\
& \left(\beta=e^{i 2 k \pi}, k \rightarrow 0 \text { for } w \rightarrow 0, \alpha_{1}=e^{i k \pi}, \alpha_{2}=-e^{-i k \pi}, \alpha_{3}=-e^{i k \pi}, \alpha_{4}=e^{-i k \pi}\right)
\end{aligned}
$$

The above function maps the region outside the area $A_{1} A_{2} A_{3} A_{4}$ (see Fig.1b) to the region outside $\gamma$ in the mathematical plane. In other words, one introduces Eq.(1) into the already available solution $[4,10]$ of the circular ring of outer radius $\mathrm{R}_{\mathrm{O}}$. Then, demanding that the rectangular notch $\mathrm{A}_{1} \mathrm{~A}_{2} \mathrm{~A}_{3} \mathrm{~A}_{4}$ must be free from stresses, and assuming, also, that the notch has too little affection on the boundary of the disc, the solution of the problem is sought in the form:

$$
\varphi(z)=\varphi_{o}(z)+\varphi_{*}(z) ; \quad \psi(z)=\psi_{o}(z)+\psi_{*}(z)
$$

where $\varphi_{o}(z)$ and $\psi_{o}(z)$ are given by the following expressions:

$$
\begin{aligned}
& \varphi_{o}(z)=\frac{P_{c}}{\pi}\left\{b_{0} z+\frac{B_{2}}{3} z^{3}-B_{-2} z^{-1}+\sum_{n=1}^{\infty}\left[\frac{B_{4 n}}{4 n+1} z^{4 n+1}-\frac{B_{-4 n}}{4 n-1} z^{-(4 n-1)}+\frac{B_{2(2 n+1)}}{4 n+3} z^{4 n+3}-\frac{B_{-2(2 n+1)}}{4 n+1} z^{-(4 n+1)}\right]\right\} \\
& \psi_{0}(z)= \\
& \frac{P_{c}}{\pi}\left\{B_{0}^{\prime} z+\frac{B_{2}^{\prime}}{3} z^{3}-b_{-2}^{\prime} z^{-1}-\frac{B_{-4}^{\prime}}{3} z^{-3}+\sum_{n=1}^{\infty}\left[\frac{B_{4 n}^{\prime}}{4 n+1} z^{4 n+1}+\frac{B_{2(2 n+1)}^{\prime}}{4 n+3} z^{4 n+3}-\frac{B_{-4(n+1)}^{\prime}}{4 n+3} z^{-(4 n+3)}-\frac{B_{-2(2 n+1)}^{\prime}}{4 n+1} z^{-(4 n+1)}\right]\right\}
\end{aligned}
$$

providing the solution of the circular ring, as it is analytically described by Kourkoulis et al. [12]. In the above expressions $\varphi_{*}(z)$ and $\psi_{*}(z)$ are analytic functions perturbing the ring's solution due to the introduction of the notch.

Alternatively, instead of the ring the intact disc could have been used as the solution basis. In this direction, it is assumed that the inner radius of the ring tends to zero. Combining Eqs.(1)-(3) and introducing them in the boundary condition for zero stresses on the rectangular notch:

$$
\varphi(s)+\frac{\omega(s)}{\overline{\omega^{\prime}(s)}} \overline{\varphi^{\prime}(s)}+\overline{\psi(s)}=0
$$

(where s is the point $\zeta$ on $\gamma$ ) one obtains (after some relatively lengthy algebra) the quantities $\varphi_{*}(₹)$ and $\psi_{*}(₹)$ solving the problem [10]. The configuration of this alternative problem is shown in Fig.2, where, in accordance with the solution of the circular ring, the disc with the notch is to be subjected to a parabolic pressure along two symmetric arcs of its periphery. In the same figure the introduction of the curvilinear coordinate system $\varrho=$ const., $\theta=$ const., at any point of the disc with the notch is shown, as it is dictated by the mapping function described in Eq.(1). 

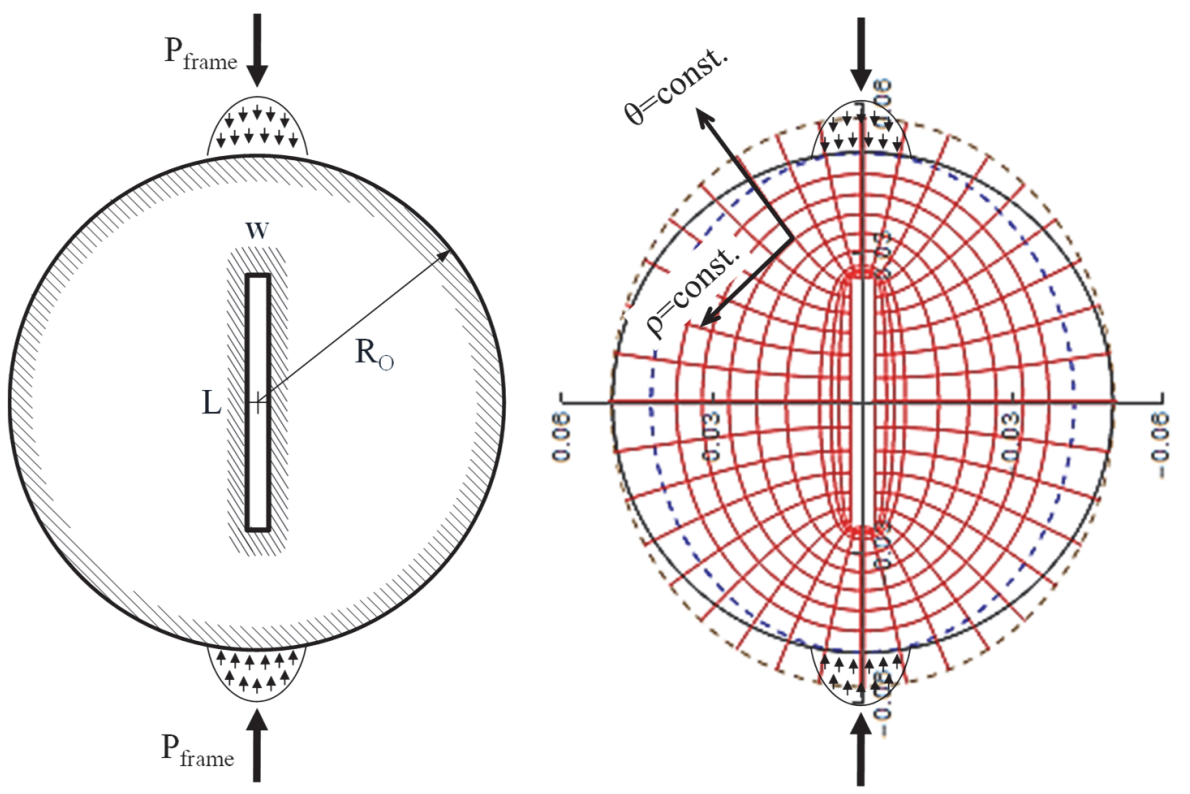

Figure 2: The notched disc under a parabolic distribution of radial stresses along two symmetric finite arcs of its perimeter (left) and the curvilinear coordinate system used in the analytic solution (right).

Then, stresses and displacements at any point of the disc are obtained using the well-known expressions [9]:

$$
\begin{aligned}
& \sigma_{\rho}-i \sigma_{\rho \theta}=2 \Re \Phi(\zeta)-\frac{e^{2 i \theta}}{\overline{\omega^{\prime}(\zeta)}}\left[\overline{\omega(\zeta)} \Phi^{\prime}(\zeta)+\omega^{\prime}(\zeta) \Psi(\zeta)\right], \quad \sigma_{\rho}+\sigma_{\theta}=4 \Re \Phi(\zeta) \\
& 2 \mu(u+i v)=\kappa \varphi(\zeta)-\frac{\omega(\zeta)}{\overline{\omega^{\prime}(\zeta)}} \overline{\varphi^{\prime}(\zeta)}-\overline{\psi(\zeta)}, \quad\left(\Phi(\zeta)=\frac{\varphi^{\prime}(\zeta)}{\omega^{\prime}(\zeta)}, \Psi(\zeta)=\frac{\psi^{\prime}(\zeta)}{\omega^{\prime}(\zeta)}\right)
\end{aligned}
$$

Using these formulae, the deformed configuration of the notch, as well as the stress-field components are determined. The deformed shape of the notch and the variation of the stresses along characteristic loci of the notched disc will be considered in juxtaposition to the respective numerical results that are obtained according to the analysis of the following section.

As a typical example, highlighting the capabilities of the above-described analytic approach, the transverse stress $\sigma_{\theta}$ (normalized over the amplitude, $\mathrm{P}_{c}$, of the parabolic distribution of radial stresses exerted on the disc) is plotted in Fig.3, along a part of the perimeter of the notch, which corresponds to the $\left[0^{\circ}, 60^{\circ}\right]$ interval of the angle $\theta$ in the $\zeta$-plane and the $\theta=$ const. hyperbolas in the z-plane (Fig.3a). Two cases are studied, one corresponding to a relatively wide notch, i.e., with $\mathrm{w}=5 \mathrm{~mm}$ (Fig.3b) and one corresponding to a relatively narrow one, i.e., with $\mathrm{w}=0.5 \mathrm{~mm}$ (Fig.3c). The crucial role of the parameter $\mathrm{w}$ is obvious by simply comparing Fig.3b and Fig.3c. Indeed, for the wider notch the normalized values of the $\sigma_{\theta}$ stress component vary in the $\left(-0.80 \mathrm{P}_{c}, 0.85 \mathrm{P}_{c}\right)$ interval. On the other hand, for the narrower notch the respective values of the $\sigma_{\theta}$ stress component vary in the $\left(-0.50 \mathrm{P}_{\mathrm{c}}, 5.20 \mathrm{P}_{\mathrm{c}}\right)$ interval. The maximum value of $\sigma_{\theta}$ in the case of the narrow notch is more than six times higher compared to that of the wider notch. It is obvious that ignoring the role of w (by assuming, for example, that the notch resembles a mathematical crack, for which w tends to zero, an assumption that is impossible to be realized for practical reasons) definitely undermines the validity of the outcomes of the standardized techniques employed for the determination of the fracture toughness, $\mathrm{K}_{\mathrm{IC}}$.

\section{NUMERICAL STUDY}

$\mathrm{T}$ he formulae provided by the analytic solution have the advantage of being full-field (i.e., they provide the stressand displacement-field components at any point of the notched disc) and of closed form, however they are quite lengthy. Therefore, employing them in exhaustive parametric analyses is rather tedious. Moreover, the fact that before applying these formulae, convergence issues of the series expansions have to be carefully considered, renders their 


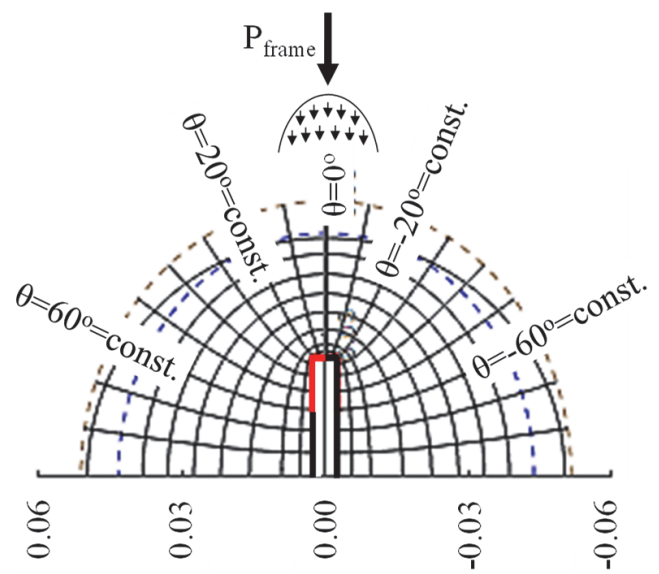

(a)

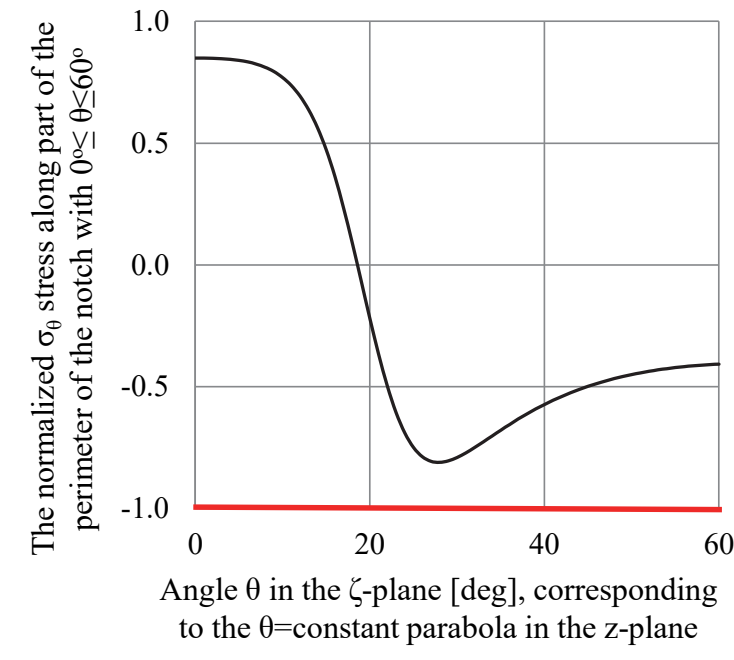

(b)

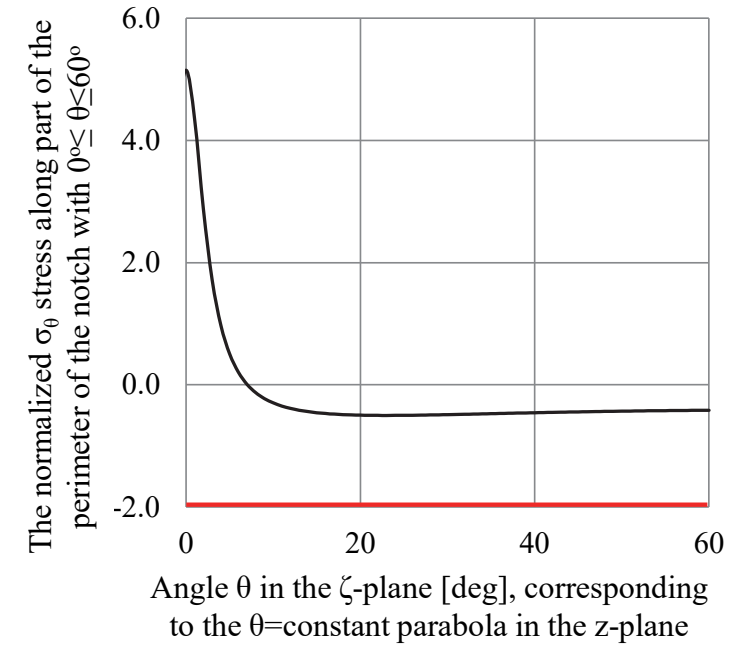

(c)

Figure 3: (a) The locus along which the stresses are plotted (red line); (b, c) The variation of the transverse stress $\sigma_{\theta}$ (normalized over the amplitude, $\mathrm{P}_{\mathrm{c}}$, of the parabolic distribution of radial stresses exerted on the disc) along the part of the perimeter of the notch shown in Fig.3a, for $\mathrm{w}=5 \mathrm{~mm}$ (b) and $\mathrm{w}=0.5 \mathrm{~mm}$ (c).

practical use inflexible and time consuming. In this direction, it was thought that one could take advantage of the analytic formulae in order to validate a numerical model, which, in turn, could be used for thorough parametric investigation of the geometric factors. In this context, a three-dimensional numerical model is designed using the finite elements method and employing the commercially available software ANSYS-19, as it is described in next sections.

\section{Reference numerical model}

A disc of diameter equal to $100 \mathrm{~mm}$ and thickness equal to $10 \mathrm{~mm}$, with a central notch of $50 \mathrm{~mm}$ length and $5 \mathrm{~mm}$ width, was chosen as the reference model. The radius of curvature at the notch's corners was set equal to $0.33 \mathrm{~mm}$. The disc was assumed to be squeezed between the curved loading platens suggested by the ISRM standard. The whole complex consisting of the disc and the two loading platens was modelled (Fig.4), in an attempt to approach the actual loading scheme developed along the disc-loading platen interface (without any additional assumption for this issue).

The disc was considered to be made of plexiglass (modulus of elasticity and Poisson's ratio equal to 3.2 GPa and 0.36, respectively) while the mechanical properties of steel (modulus of elasticity and Poisson's ratio equal to 210 GPa and 0.30 , respectively) were assigned to the loading platens. The coefficient of friction between the plates and the disc was set equal to 0.01 , in order to approach the assumptions adopted in the analytic solution, where the role of friction was ignored.

The volumes of the model were meshed using the SOLID185 element while the TARGE170 and CONTA173 elements were used for the two material interfaces between the platens and the disc. The final meshing (Fig.5) was decided based on a thorough convergence analysis carried out as a first step of the numerical study. 
The nodes of the base of the lower platen were rigidly clamped while the nodes of the lateral surfaces of both platens were not permitted to move along the horizontal x-axis. Finally, a vertical downwards displacement equal to $2.275 \mathrm{~mm}$ was imposed on the nodes of the upper surface of the upper platen (Fig.6). The specific displacement value corresponds to the $20 \mathrm{kN}$ load applied in the analytic solution used for the validation of the numerical model.

\section{$V$ alidating the numerical model taking advantage of the analytical solution}

Due to the double symmetry of the model, the results obtained by the numerical model and those provided by the analytic solution will be presented only for one quarter of the disc-loading platens complex. More specifically, in Fig.7a the initial shape of one quarter of the perimeter of the notch (black line) is drawn, in juxtaposition to its deformed shape, as it was obtained by the analytical (red line) and the numerical (blue line) solutions. The difference between them does not exceed $4 \%$ at any point of the locus considered, which is obviously well accepted. Even, this small difference may be explained by the fact that the series expressions of the analytic solution used for the calculation of the displacements are too complicated imposing limitations on the number of terms that can be used.
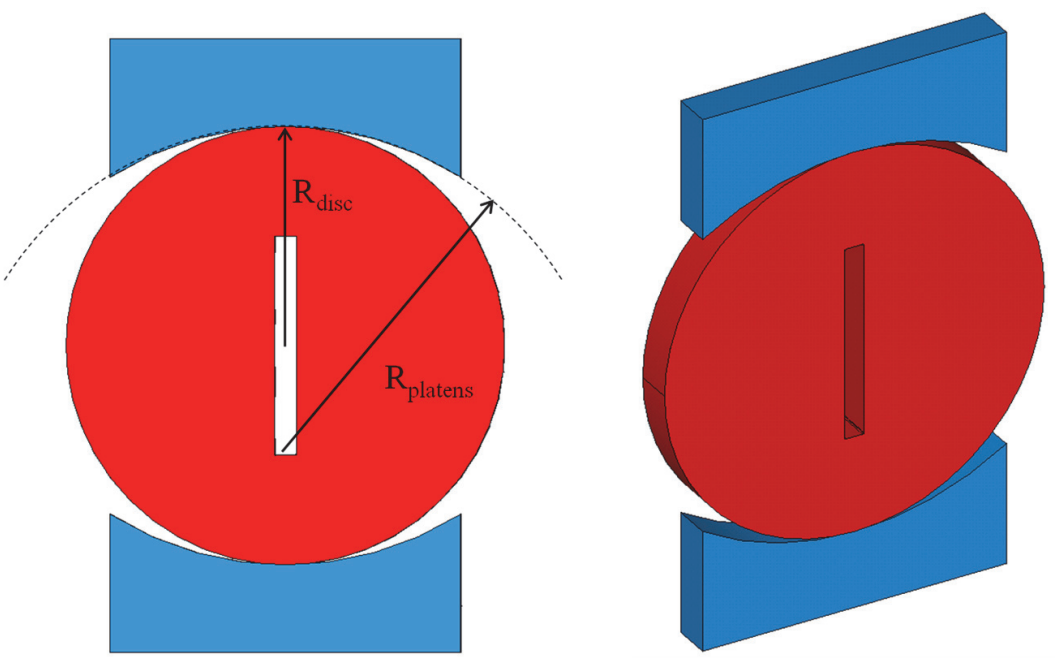

Figure 4: The reference numerical model.

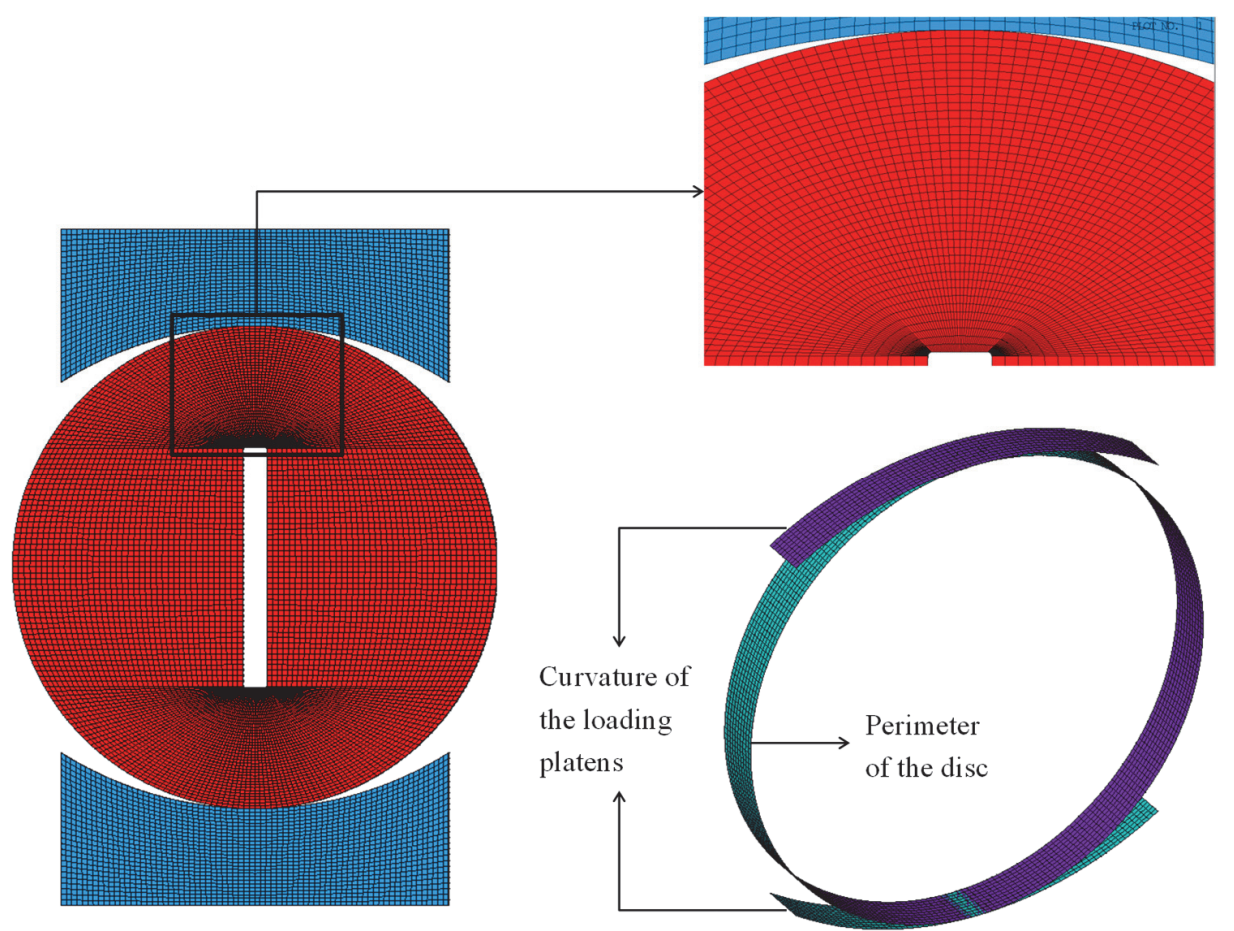

Figure 5: The meshing of the reference model and the material interfaces. 


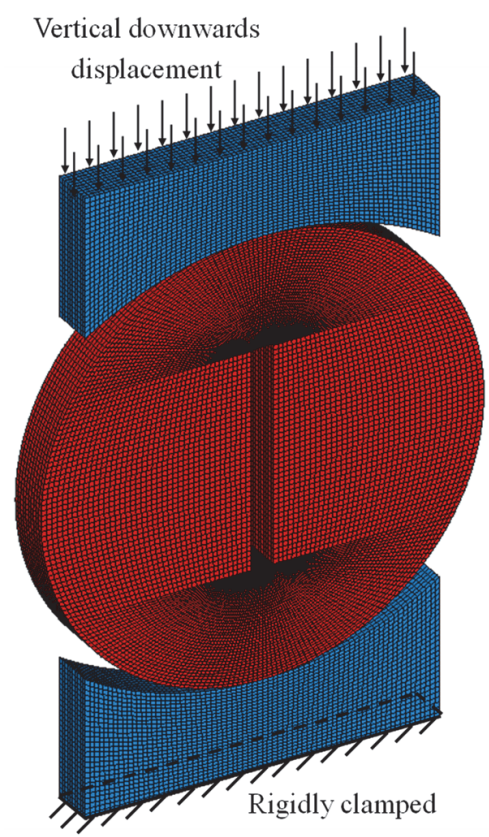

Figure 6: The loading and the boundary conditions.
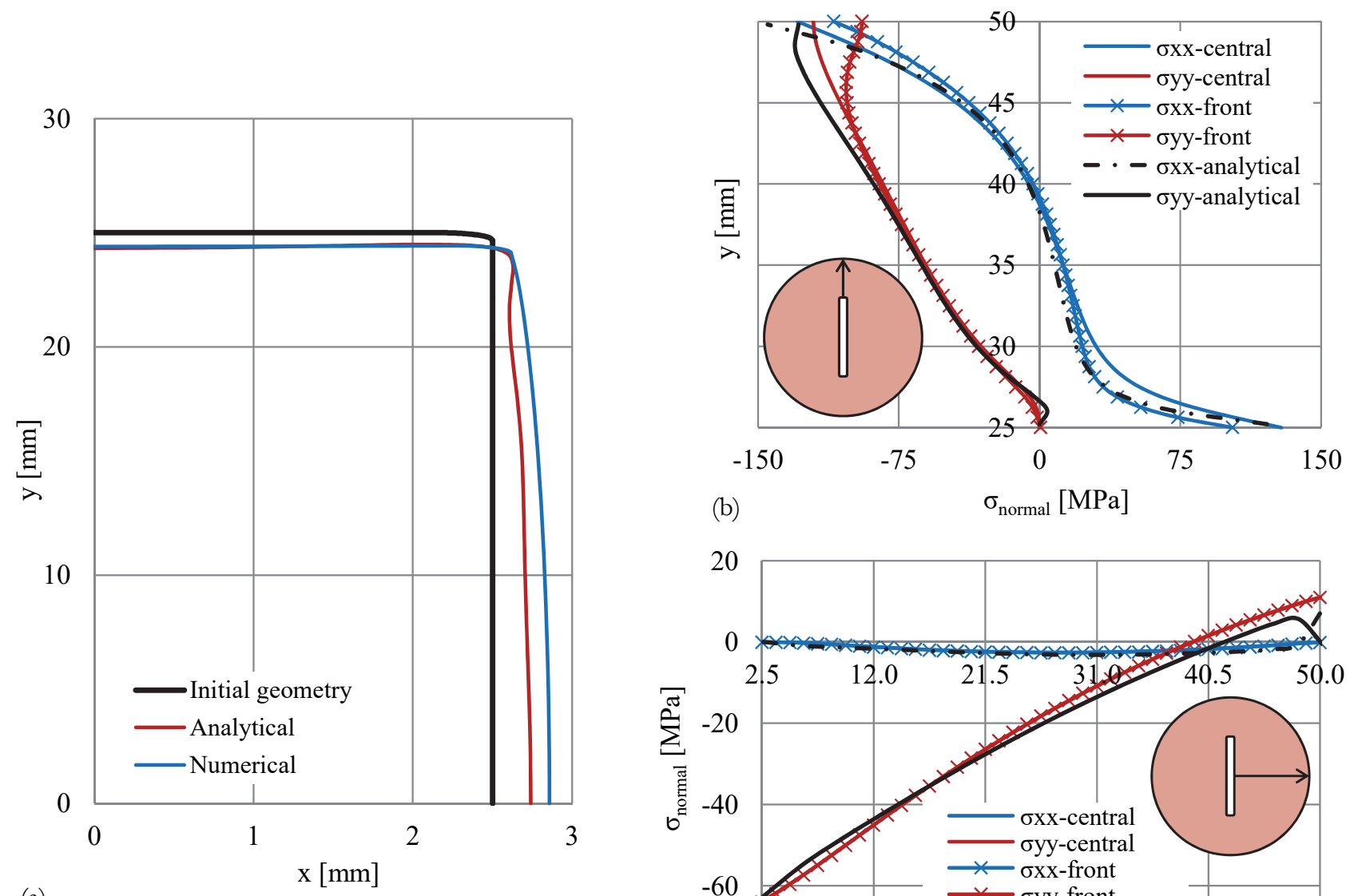

(a)

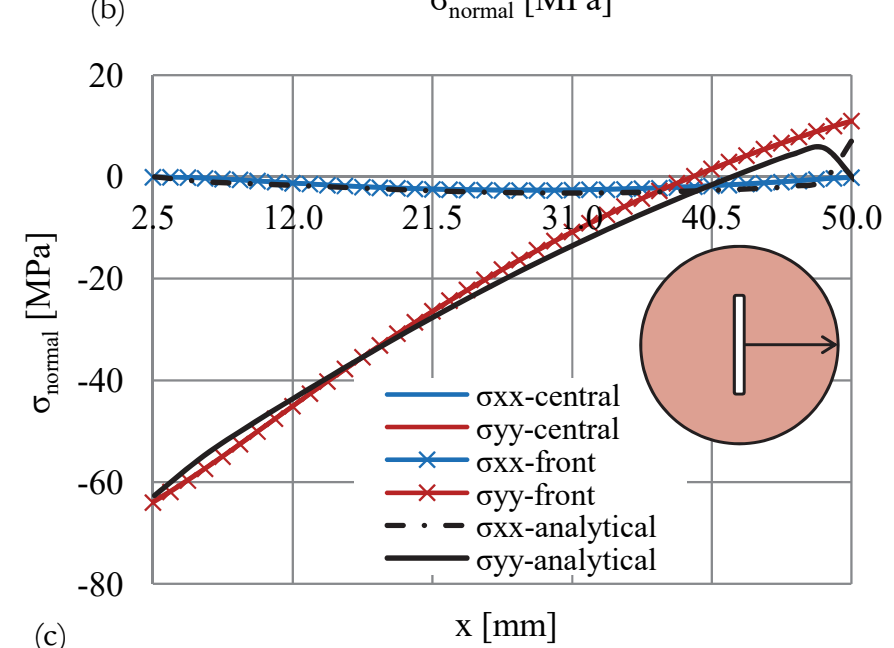

Figure 7: (a) Undeformed and deformed geometries of one quarter of the perimeter of the notch; normal stresses developed along the vertical $y$-axis (b) and the horizontal $x$-axis (c), as they were obtained by both the analytical and the numerical approaches. 
Concerning now the stress field components, it is here recalled that the numerical model deals with the problem in three dimensions (contrary to the analytic solution which is by default two dimensional). In this context, the normal stresses developed both at the central cross section of the disc as well as these developed within the plane of its front face are here taken into account. The comparison between numerical and analytical data is seen in Fig. $7 \mathrm{~b}$ for the normal stresses which are developed along the vertical y-axis (i.e., the line of symmetry along the longitudinal axis of the notch) and in Fig.7c for the normal stresses developed along the horizontal x-axis (i.e., the line of symmetry normal to the longitudinal axis of the notch). It is clearly seen from these figures that non-ignorable differences between the data of the analytic and numerical solutions appear only as one approaches the immediate vicinity of the disc-loading platens contact arc. On the contrary, in the immediate vicinity of the crown of the notch (i.e., in the area of utmost importance for the purposes of the present study) the mutual agreement is excellent. It is thus concluded that the numerical model can be safely used to study either more complicated loading schemes or for exhaustive parametric analyses of the role of various geometrical characteristics.

\section{Parametric investigation}

After its validation, the reference model was properly modified to design six additional models, in order to quantify the influence of the geometrical characteristics of the notch on the stress field developed in the disc. The modified geometries of the discs are presented in Fig.8 (the loading platens are not shown in this figure, although the whole disc-loading platens complex is again simulated, as it was the case for the reference model). The radius of curvature of the corners of the notch, and the length and width of the notch were the characteristics considered and the values assigned to each one of them are shown in Table 1. Moreover, the role of the third dimension, namely that along the disc's thickness was, also, studied.

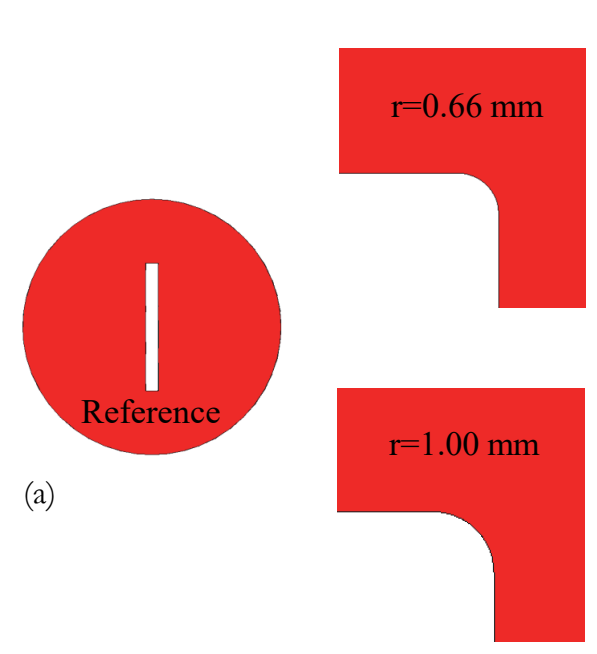

(b)

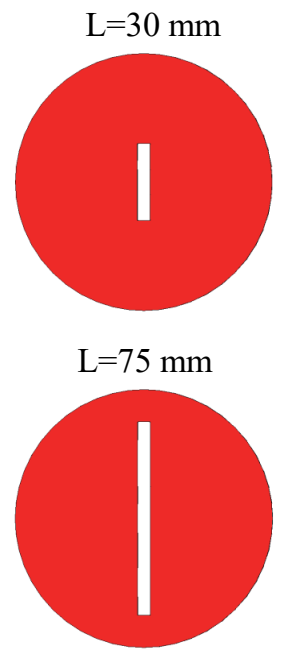

(c)
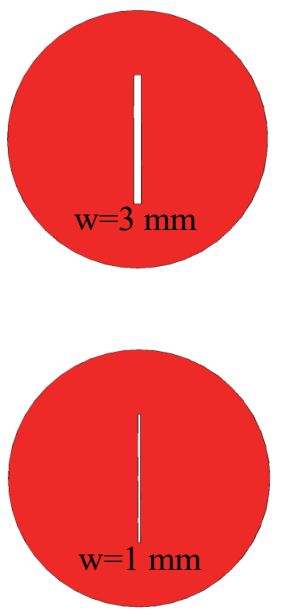

(d)

Figure 8: The numerical models (only the discs are presented here) of the parametric analysis. (a) The reference disc, (b) enlarged views of one of the corners of the notch (parameter r); (c) the discs for the parameter $\mathrm{L}$ and (d) the discs for the parameter w.

\begin{tabular}{cccc}
\hline $\begin{array}{c}\text { Model } \\
\text { Reference model }\end{array}$ & $\begin{array}{c}\text { Radius of the corners of the notch, r } \\
{[\mathrm{mm}]}\end{array}$ & $\begin{array}{c}\text { Length of the notch, L } \\
{[\mathrm{mm}]}\end{array}$ & $\begin{array}{c}\text { Width of the notch, w } \\
{[\mathrm{mm}]}\end{array}$ \\
$\mathrm{r}=0.66 \mathrm{~mm}$ & $\mathbf{0 . 6 6}$ & 50 & 5 \\
$\mathrm{r}=1.00 \mathrm{~mm}$ & $\mathbf{1 . 0 0}$ & 50 & 5 \\
$\mathrm{~L}=30 \mathrm{~mm}$ & 0.33 & 50 & 5 \\
$\mathrm{~L}=75 \mathrm{~mm}$ & 0.33 & $\mathbf{3 0}$ & 5 \\
$\mathrm{w}=1 \mathrm{~mm}$ & 0.33 & $\mathbf{7 5}$ & $\mathbf{1}$ \\
$\mathrm{w}=3 \mathrm{~mm}$ & 0.33 & 50 & $\mathbf{3}$ \\
\hline
\end{tabular}

Table 1: The parameters considered during the numerical analysis and the values assigned to each model. 


\section{RESULTS}

\section{The stress field for the reference model and the role of the third dimension}

he distribution of the equivalent stress developed in the whole complex is presented in Fig.9a. As it was expected, the most severely stressed areas are those in the immediate vicinity of the rounded corners of the notch. In addition, it is seen that the equivalent stress attains lower values as one is directed from the face of the disc towards its central plane. Plotting the equivalent stress along one quarter of the notch, the previously mentioned comment is more clearly seen (Fig.9b). The first point of the plots (position at $0 \mathrm{~mm}$, Point $\mathrm{M}$ ) corresponds to the midpoint of the width of the notch and the last one (position at $28 \mathrm{~mm}$, Point $\mathrm{K}$ ) corresponds to the midpoint of the notch's length (clockwise direction). For clarity, the locus considered was divided into three segments (embedded sketch in Fig.9(b2)), i.e., the horizontal segment or in other words the one along the x-axis (continuous line), the corner of the notch (dashed line) and the vertical one or in other words the segment which is parallel to the y-axis (dotted line). The same line code was used for the stresses, for which, in addition, blue colour indicates the stresses developed at the central cross section of the disc while the red one the stresses at its front face. It is seen from Fig. $9 \mathrm{~b}$ that the maximum equivalent stress at the front surface of the disc is about $15 \%$ larger than the respective value at the central cross section. It is, also, interesting to note that the equivalent stress at the front surface of the disc becomes significantly larger from the equivalent stress at the central cross section only at the immediate vicinity of the corner of the notch.
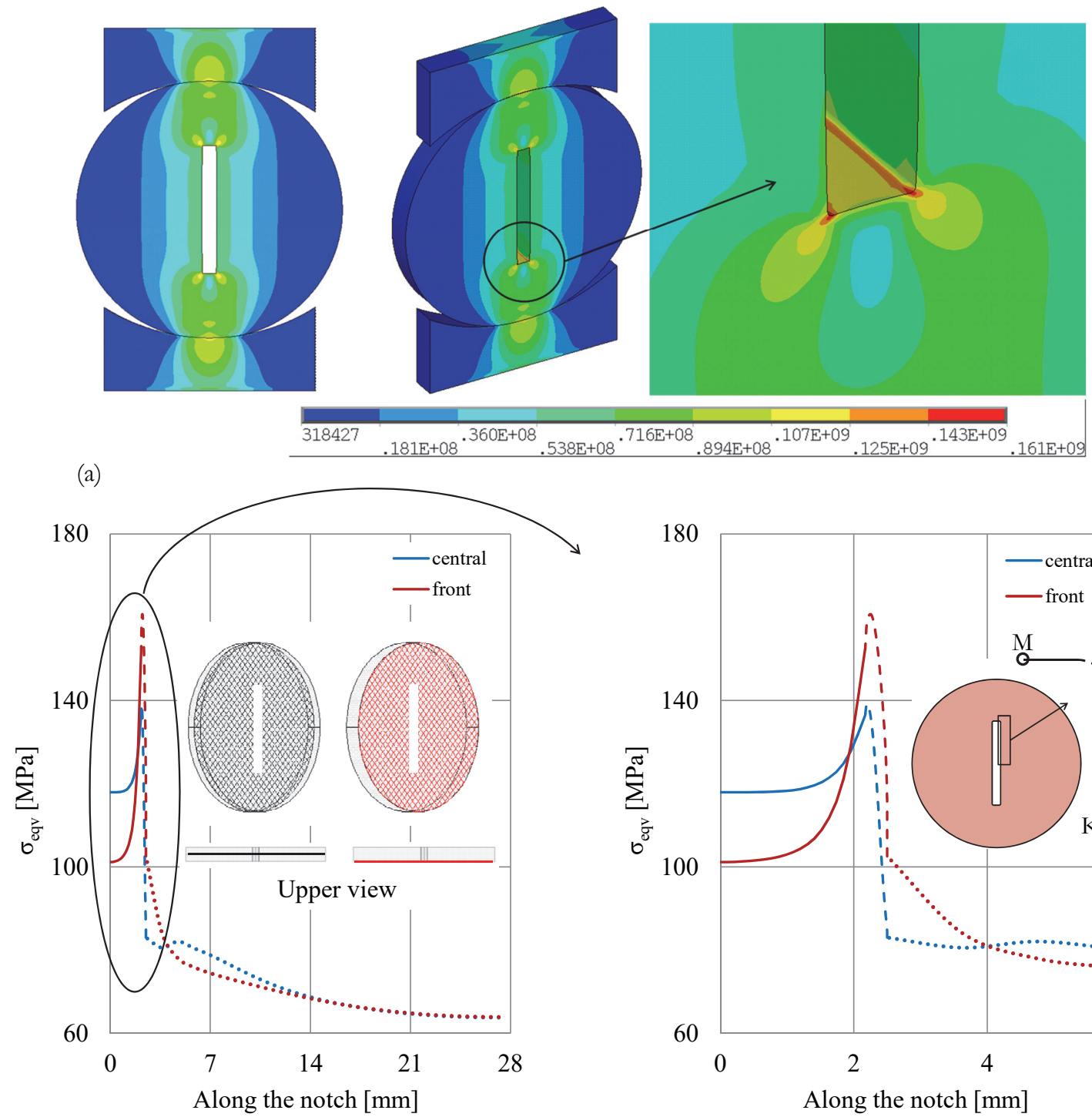

(b1)

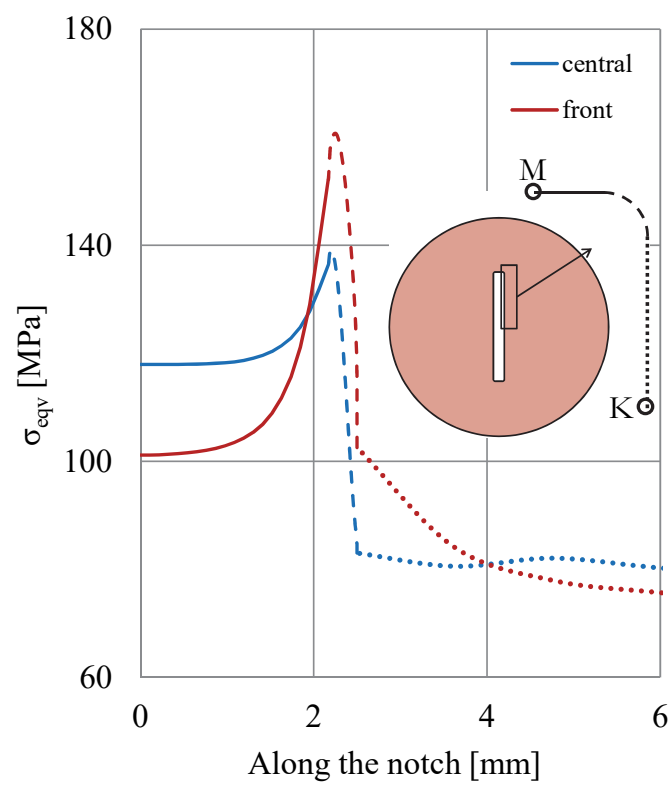

Figure 9: Reference model. (a) The overall distribution of the equivalent stress in [Pa] over the disc-loading platens complex; (b1) its variation along one quarter of the perimeter of the notch and (b2) a detailed view around the corner of the notch. 
Contrary to the equivalent stress, the maximum principal stress, $\sigma_{1}$, seems to attain larger values as one is directed from the face of the disc towards its center, as it is seen from Fig.10a. A more conceivable interpretation of the differences of the maximum principal stresses, $\sigma_{1}$ over the plane of the front face (red colour) and over that of the central cross section (black colour) is depicted in Fig.10b. In this figure one quarter of the notch was again isolated, due to the double symmetry of the model, and the stress developed at each point of the notch is drawn (for clarity reasons, the numerical values of $\sigma_{1}$, attained at some strategic points of the locus are added in the figure). As it was expected, the maximum principal stress along the length of the notch (free boundary) equals zero. Based on this figure, it can be stated that, contrary to the equivalent stress, the maximum principal stress is significantly larger at the central cross section (black colour) along the major part of the perimeter of the notch. Only along the notch's rounded corners the two plots tend to coincide, or in other words the role of the third dimension becomes negligible.

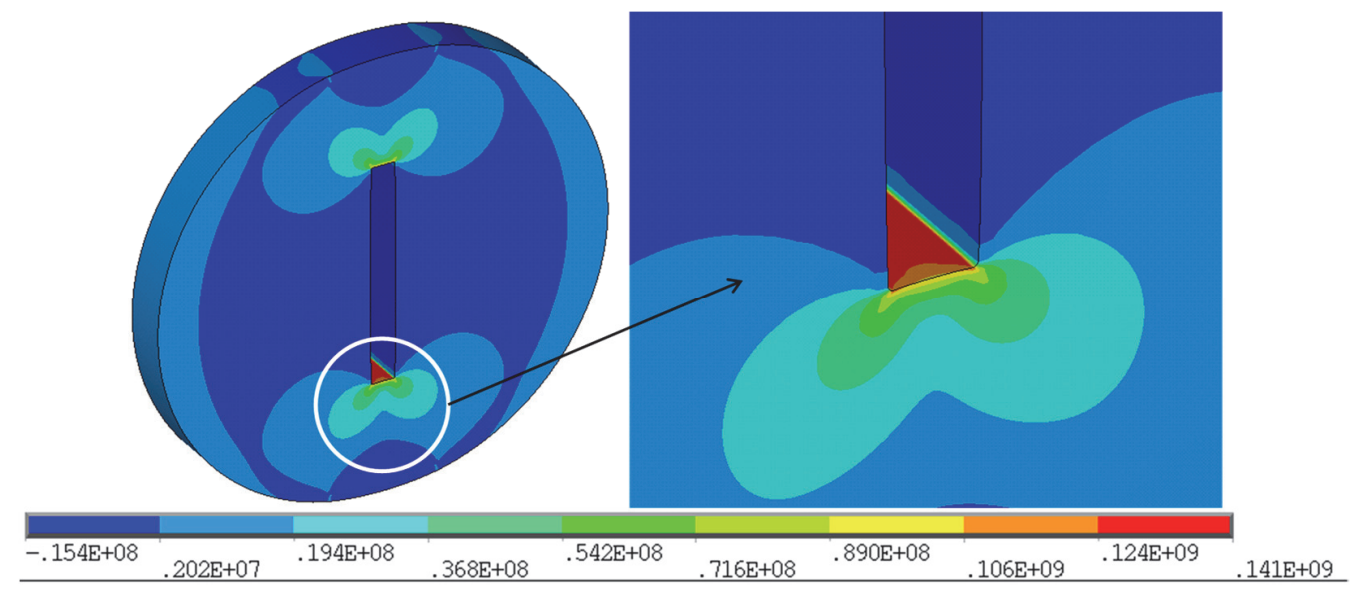

(a)

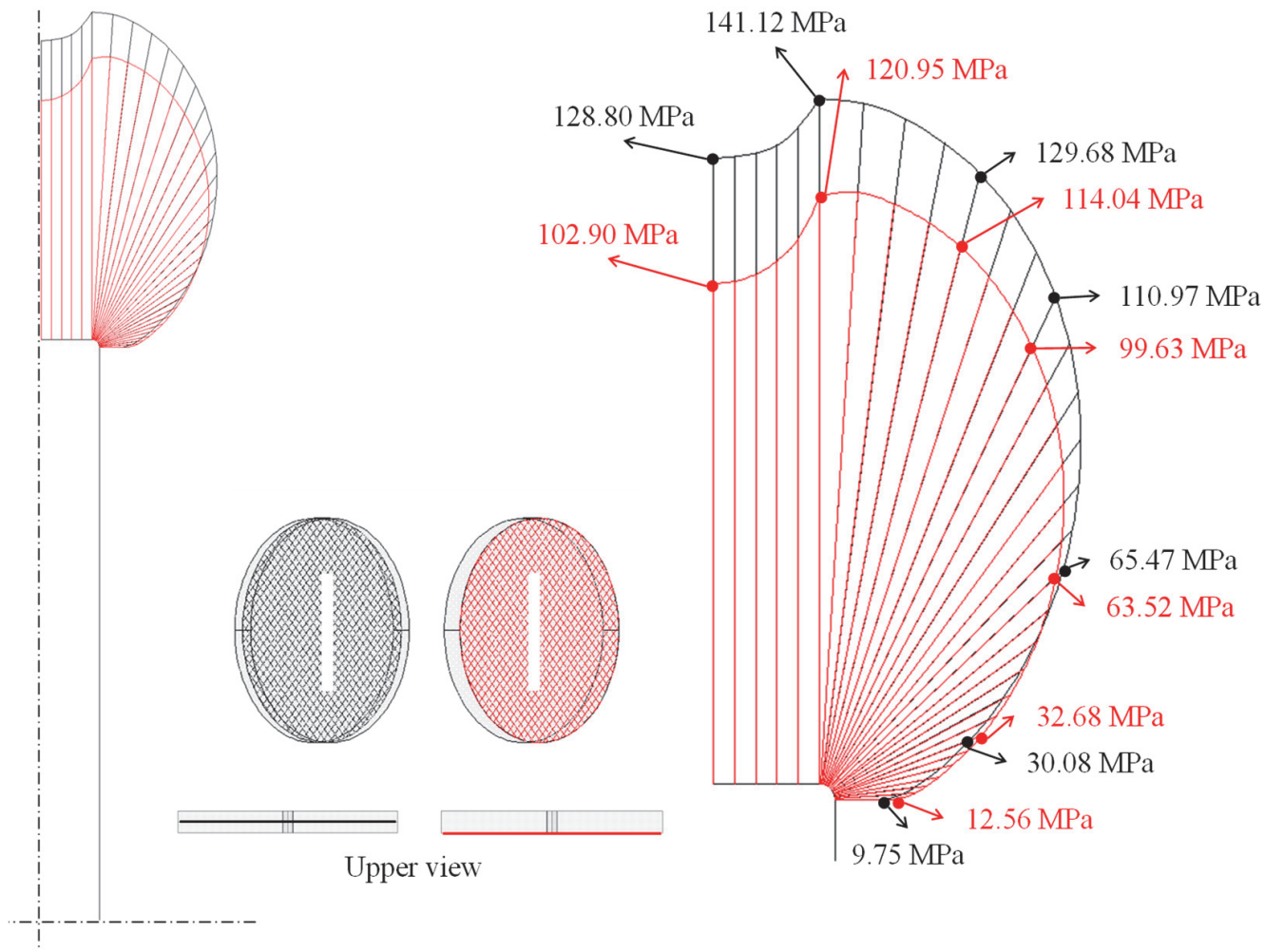

(b)

Figure 10: Reference model. (a) The distribution of the maximum principal stress in $[\mathrm{Pa}]$ and (b) its variation along one quarter of the notch over the plane of the front face (red colour) and over that of the central cross section (black colour). 
The effect of the radius of the corners of the notch

The influence of the radius of curvature at the corners of the notch on the stress field is depicted in Fig.11, in which the equivalent stress at the central cross section along the notch's perimeter is plotted for three values of $\mathrm{r}$, i.e., $\mathrm{r}=0.33 \mathrm{~mm}$, $0.66 \mathrm{~mm}$ and $1.00 \mathrm{~mm}$. It is seen that the equivalent stress developed is rather marginally influenced by the changes of $\mathrm{r}$. Indeed, even around the notch's crown, the differences detected are of the order of 5\% (Fig.11a). Regarding the maximum principal stress, $\sigma_{1}$, plotted in Fig.11b, the maximum difference is detected at the beginning of the rounded corner (point $\mathrm{N}$ in Fig.11c) and it is of the order of $15 \%$.

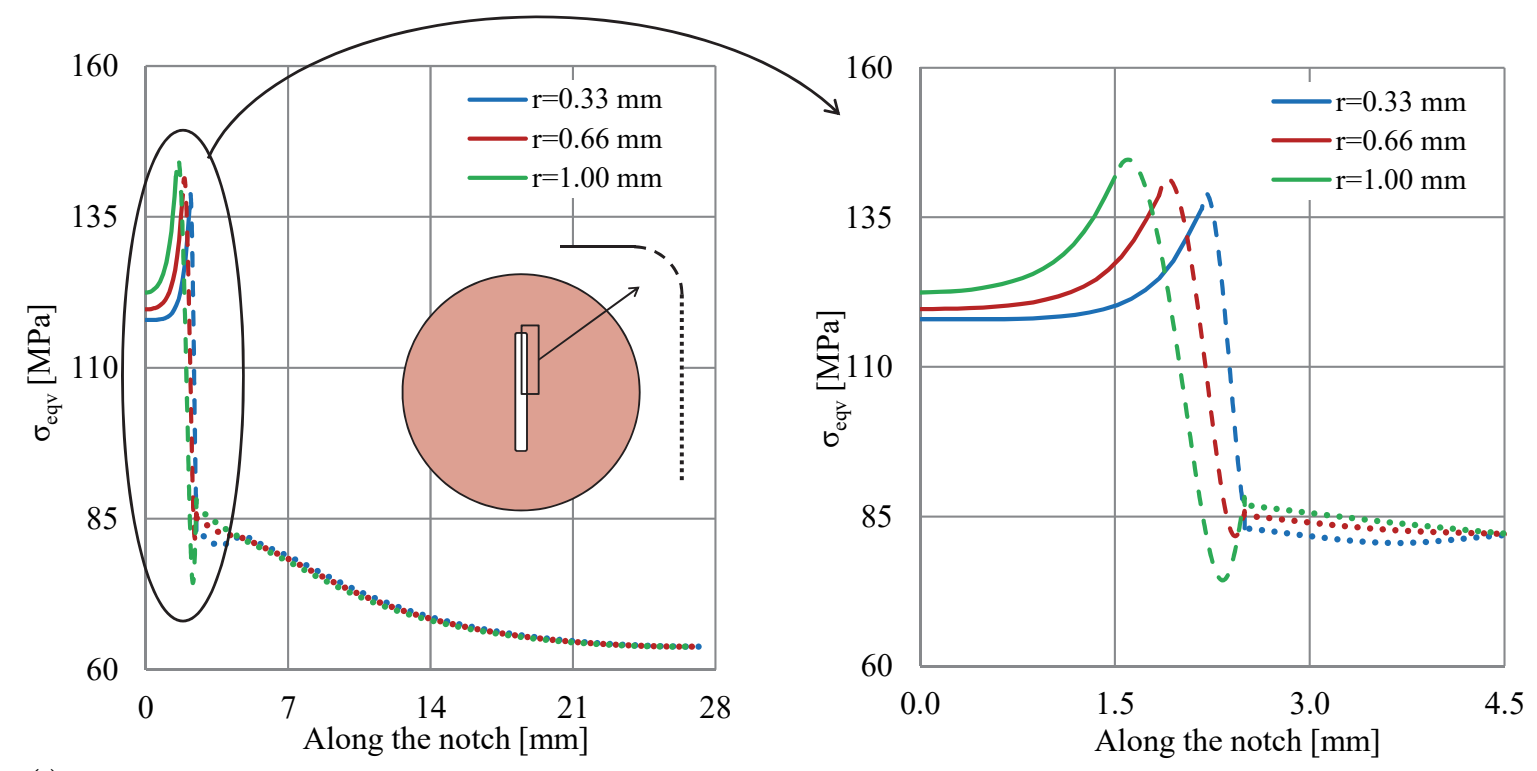

(a)

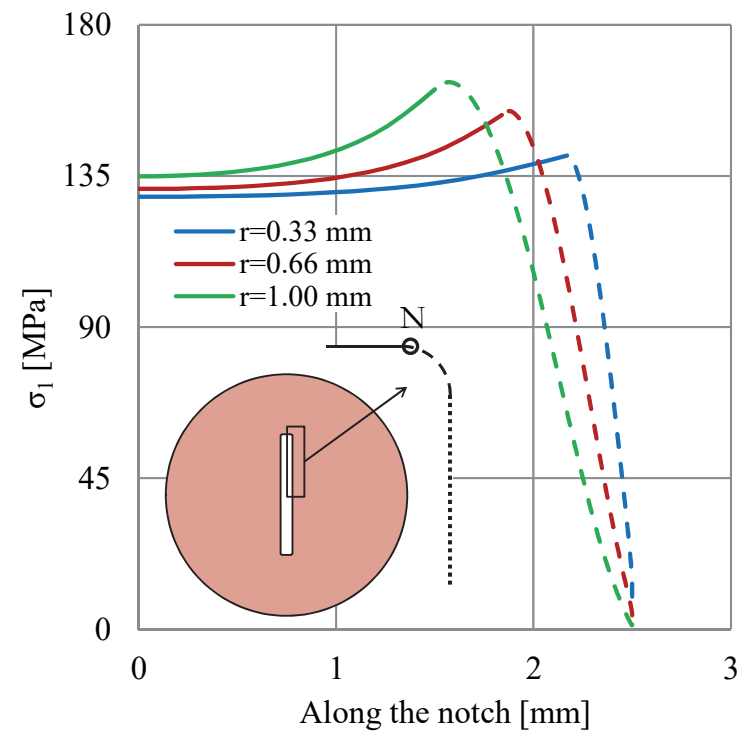

(b)

Figure 11: The role of the radius of the corners of the notch: The variation of (a) the equivalent stress and an enlarged view of the vicinity around the corner of the notch and (b) the maximum principal stress along one quarter of the notch.

\section{The effect of the width of the notch}

Concerning the role of the width of the notch, it can be said that the qualitative distribution of the equivalent stress, $\sigma_{\text {eqv }}$, in the body of the disc is not drastically different for the two additional cases studied here (i.e., $\mathrm{w}=1 \mathrm{~mm}$ and $\mathrm{w}=3 \mathrm{~mm}$ ), in comparison with the reference model with $\mathrm{w}=5 \mathrm{~mm}$. Indeed, the distributions of $\sigma_{\text {eqv }}$ are quite similar to that presented in Fig.9a for the reference model. From a quantitative point of view, however, things are drastically different, especially if atten- 
tion is focused on the area around the crown of the notch, namely, the locus most important for the determination of $\mathrm{K}_{\mathrm{IC}}$, taking into account that crack is expected to start propagating from a point of the notch's perimeter located in this area. In an attempt to gain better insight on the above-mentioned differences, the distribution of the equivalent stress along the upper half of the $y$-axis (i.e., the axis parallel to the symmetry axis of the externally applied stress distribution) at the central cross section of the disc is plotted in Fig.12a. It is seen from this figure that approaching the crown of the notch the equivalent stress is about $60 \%$ higher for the case of the narrow notch. On the contrary, as one is directed far from the notch's crown (i.e., moving towards the disc-loading platen contact arc), the equivalent stress is not seriously affected by the differences of the value of $\mathrm{w}$.

As far as it concerns the second strategic locus, i.e., that along the perimeter of the notch, it can be seen from Fig.12b, that the values of the equivalent stress are almost insensitive to changes of $\mathrm{w}$, along the segment of the notch that is parallel to the loading axis (see the dotted lines in Fig.12b). However, approaching the mid-point of the notch's crown the equivalent stress for the narrow notch exceeds dramatically that of the wider notches (see the continuous lines in Fig.12b).

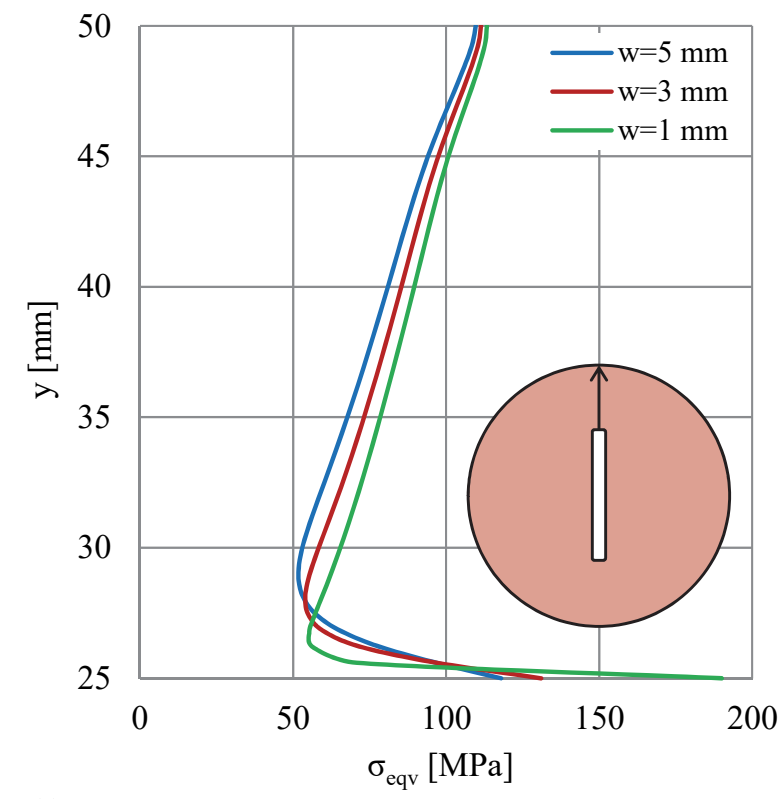

(a)

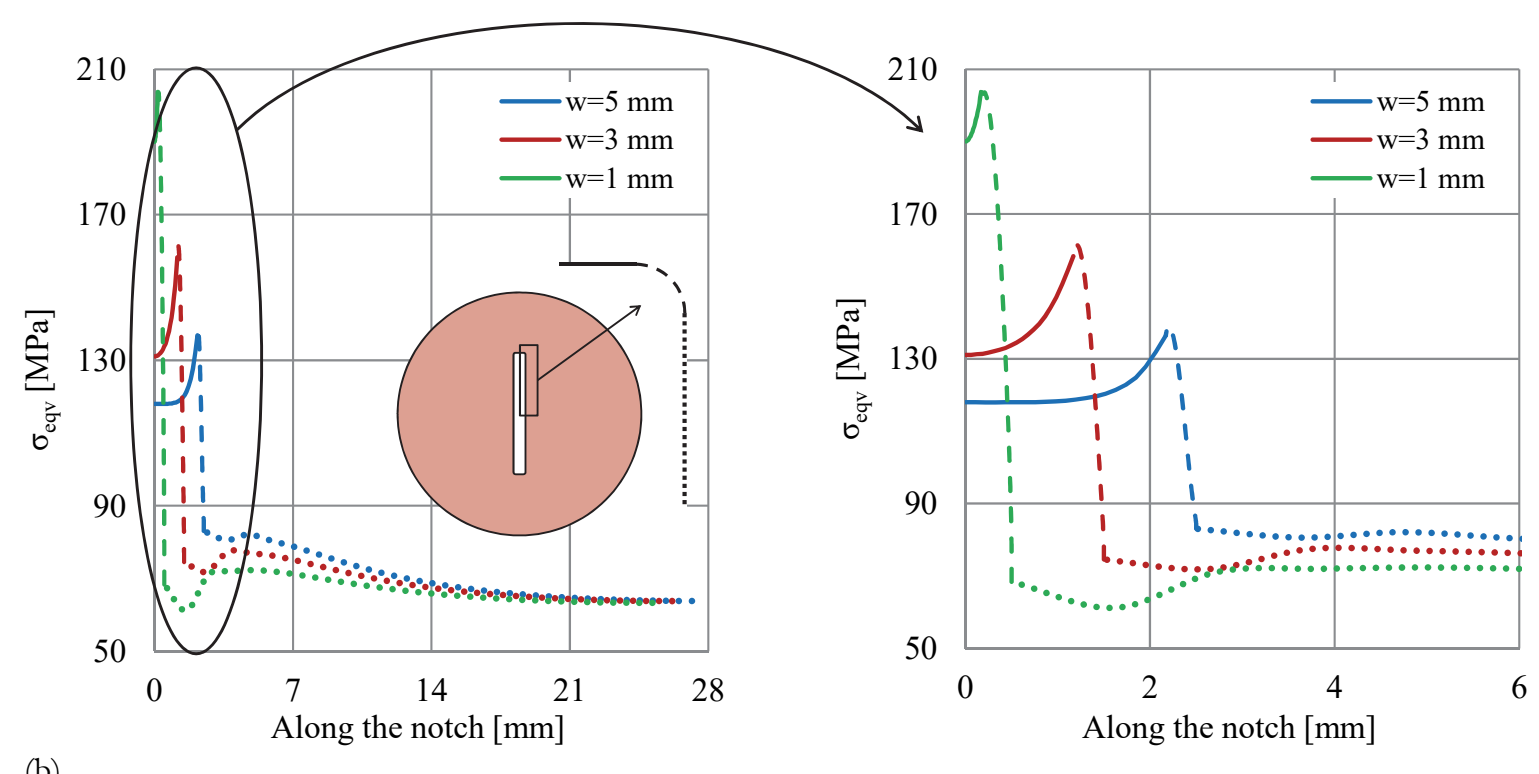

Figure 12: The role of the width of the notch: The variation of (a) the equivalent stress along the upper half of the vertical loading $y$ axis and (b) the maximum principal stress along one quarter of the notch with a detailed view around its corner. 


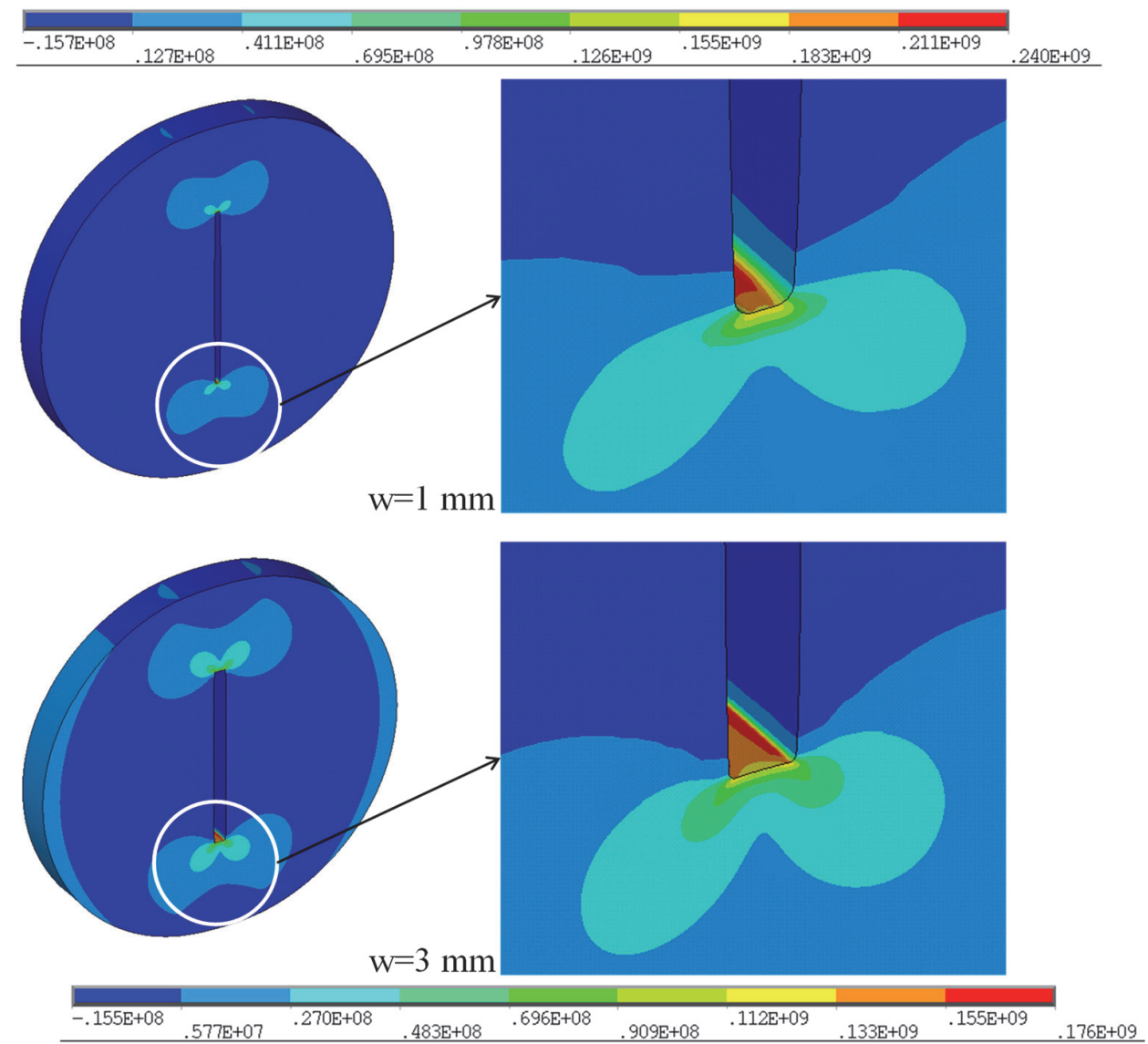

(c)

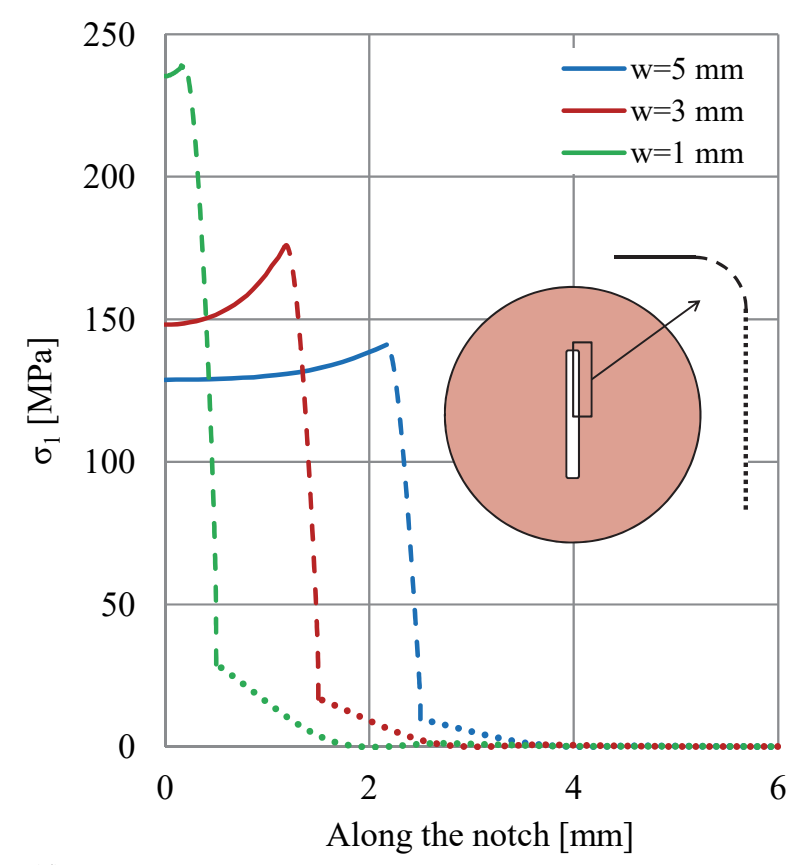

(d)

Figure 12 (continued): (c) The distribution of the maximum principal stress for the models with $\mathrm{w}=1 \mathrm{~mm}$ and $\mathrm{w}=3 \mathrm{~mm}$, (d) the variation of the maximum principal stress along one quarter of the notch.

Quite similar conclusions are drawn for the maximum principal stress, the distribution of which is plotted in Fig.12c for the cases with $\mathrm{w}=1 \mathrm{~mm}$ and $\mathrm{w}=3 \mathrm{~mm}$ (the distribution for reference case, i.e., that with $\mathrm{w}=5 \mathrm{~mm}$ has been already pre- 
sented in Fig.10a). The values of the maximum principal stress, $\sigma_{1}$, along one quarter of the notch's perimeter (again at the central cross section of the disc) are plotted in Fig.12d. Differences of the order of $70 \%$ are observed between the model with $\mathrm{w}=1 \mathrm{~mm}$ and that with $\mathrm{w}=5 \mathrm{~mm}$, indicating once again the paramount influence of the width of the notch on the stress field developed in the vicinity of the crown of the notch.

\section{The effect of the length of the notch}

The last geometrical parameter considered in this study is the length of the notch with respect to the disc's radius. Again, the distributions of the equivalent stress in the three models (i.e., the models with $\mathrm{L}=30 \mathrm{~mm}, 50 \mathrm{~mm}$ and $75 \mathrm{~mm}$ ) are qualitatively similar to each other (Fig.13a), however the differences along some characteristic paths exhibit significant quantitative differences. Indeed, the larger the length of the notch, or in other words the closer the notch's crown to the boundary of the disc, the larger the equivalent stress developed in the disc, as it can be clearly seen in Fig.13b, where the variation of the equivalent stress along the vertical y-axis of the central cross section is plotted. It is concluded from this figure that the equivalent stress developed at the midpoint of the width of the notch in case $\mathrm{L}=75 \mathrm{~mm}$ is about $60 \%$ higher compared to the respective stress developed at the same point when $\mathrm{L}=30 \mathrm{~mm}$ (Fig.13b). The difference is even larger, approximately $75 \%$, at the point where the straight segment of the width meets the starting point of the curved part of the notch' crown, as it can be seen in Fig.13c.

Differences of the same order are observed, also, for the maximum principal stress, $\sigma_{1}$. This is clearly seen in Fig.14a where the variation of the maximum principal stress along one quarter of the perimeter of the notch, at the central cross section of the disc, is shown. The difference is about $60 \%$ at the midpoint of the width of the notch and it reaches $100 \%$ at the point where the straight segment of the width meets the starting point of the curved part of the notch' crown. Even larger differences, ranging from about 25\% to more than 100\% (depending on the specific point of the locus) can be detected along the perimeter of the disc (Fig.14b).

\section{DISCUSSION AND CONCLUDING REMARKS}

T he role of some geometrical features of the notches machined in circular discs in order to experimentally determine the fracture toughness of brittle rock-like materials was studied, both analytically and numerically. The motive of the study is the difference between the configuration of the theoretical model adopted for the determination of the Stress Intensity Factor (i.e., that of the Crack Straight Through Brazilian Disc - CSTBD) and the actual configuration obtained in the laboratory, when a disc made of rock-like materials is mechanically notched. Indeed, while the theoretical model considers a relatively short "mathematical" crack (i.e., a discontinuity of short length with respect to the disc's diameter, zero distance between its lips and singular tip) the discontinuities mechanically machined are notches of finite width, without singular tip and of length well comparable to the radius of the disc.

Based on the results of a recently introduced analytical solution $[4,10]$ a numerical model was designed and validated. The calibration procedure was successful given that the results of the analytic solution and those of the numerical model are in very good agreement ignoring minor discrepancies in the immediate vicinity of the platens-disc interface (attributed to the very complex nature of the analytic solution, which renders convergence of the respective series expressions at specific loci satisfactory only when a large number of terms is used, which is inconvenient from the practical point of view). The validated model was used for a thorough parametric investigation.

The investigation highlighted the role of the third dimension (i.e., along the disc's thickness), which is usually considered of minor importance in two-dimensional studies. Differences between the stress levels at the front face of the disc and at the central vertical section approaching even $20 \%$ were detected. The role of the radius of the corners of the notch was proved to be of rather secondary importance. Variations of $r$ of the order of $200 \%$ resulted to changes of the equivalent stress lower than about $15 \%$. On the contrary, the role of the length of the notch is proven to be quite catalytic. Indeed, increasing L from 30 to 50 and $75 \mathrm{~mm}$, results to changes of the equivalent stress from 100 to 140 and to $180 \mathrm{MPa}$, respectively.

The parameter, the influence of which on the magnitude of the stress field in the immediate vicinity of the rounded corners of the notch was found to be of paramount importance is the width, w, of the artificially machined notch: Increasing w from 1 to 3 and $5 \mathrm{~mm}$ results to changes of the equivalent stress from 140 to 160 and to $200 \mathrm{MPa}$, respectively.

What is to be stressed out, is that a realistic overview of the stress field developed when a notched disc is compressed between the loading platens suggested by either ASTM or ISRM is not singular and the concept of stress intensity does not properly reflect experimental reality. In fact, it is the concept of stress concentration that is to be considered since the stress field at the crown of the notch is intensified by the presence of the notch-shaped discontinuity but by no means it approaches that of a singular field predicted by the theoretical model of the CSTBD approach. 


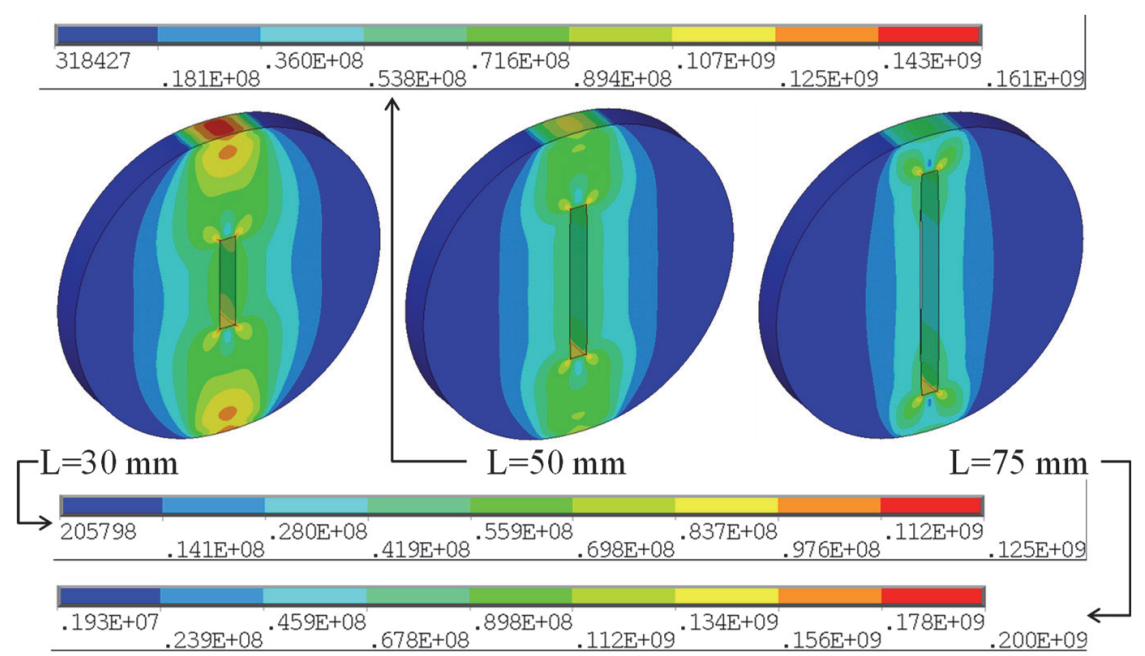

(a)

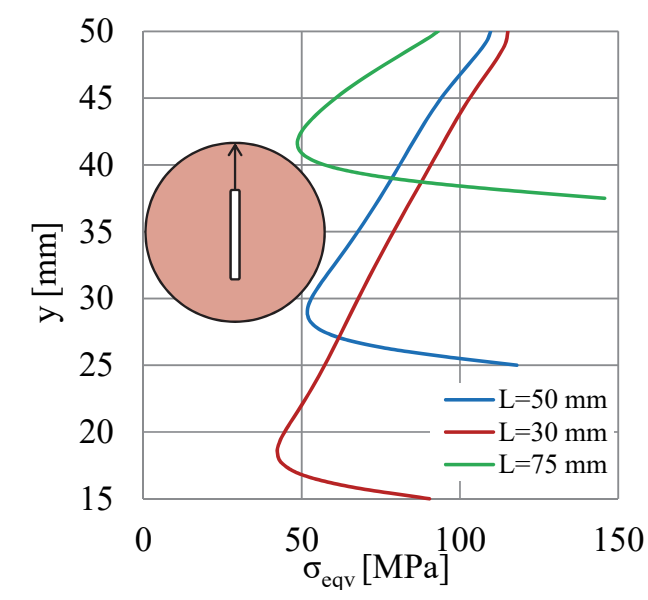

(b)
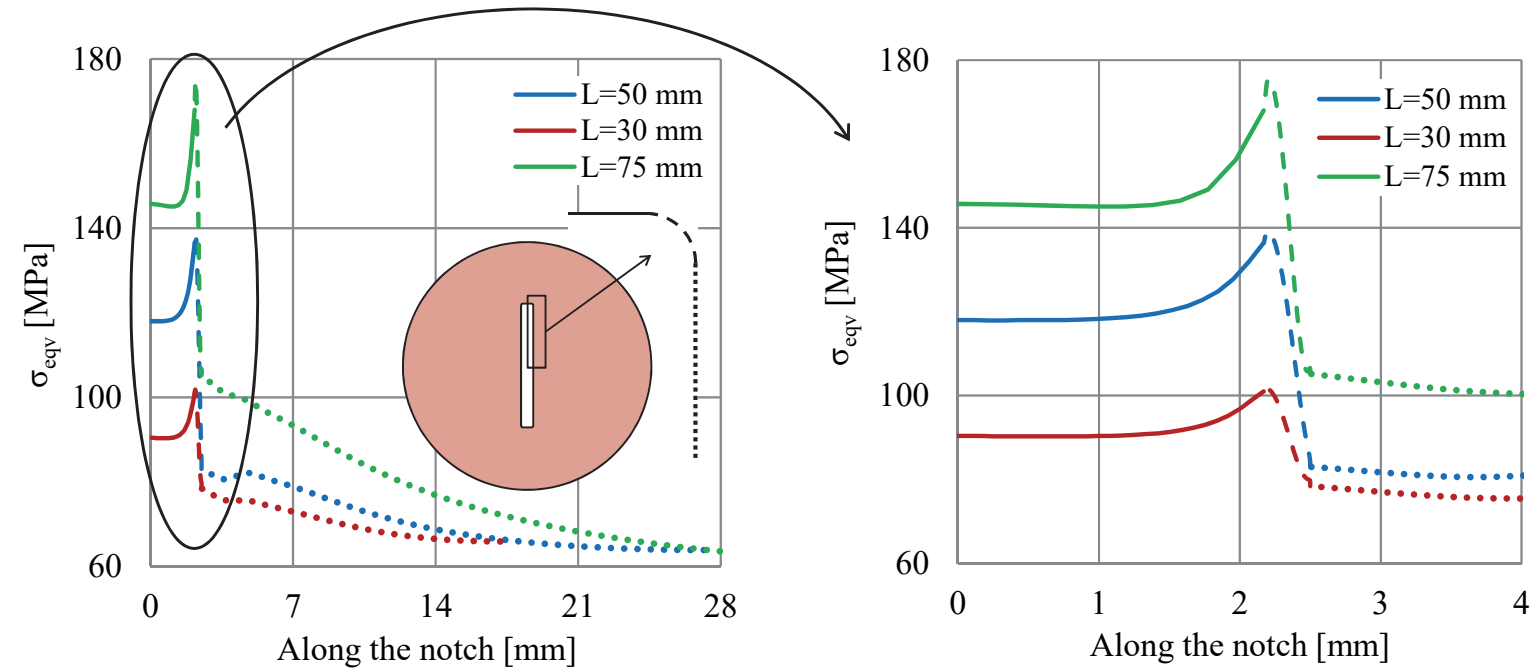

(c)

Figure 13: (a) The distribution of the equivalent stress in the disc for the three models with $\mathrm{L}=30,50,75 \mathrm{~mm}$; The variation of the equivalent stress along (b) the vertical y-axis and (c) along one quarter of the perimeter of the notch with a detailed view around its corner.

To make the above comment clearer, advantage is here taken of the analytic solution described in previous sections. The stress concentration, defined here as the ratio of the equivalent stress developed over the amplitude of the externally applied 

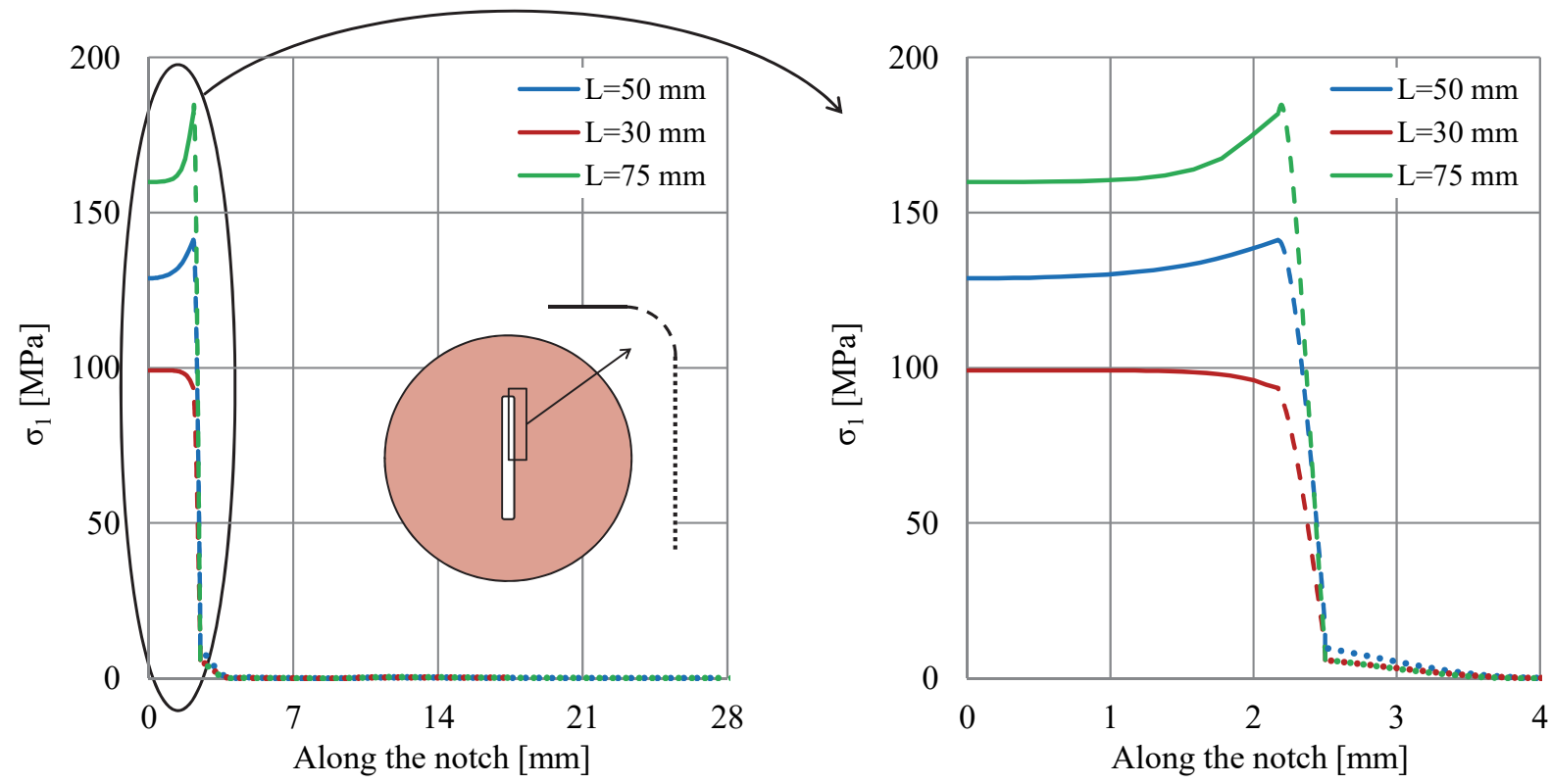

(a)

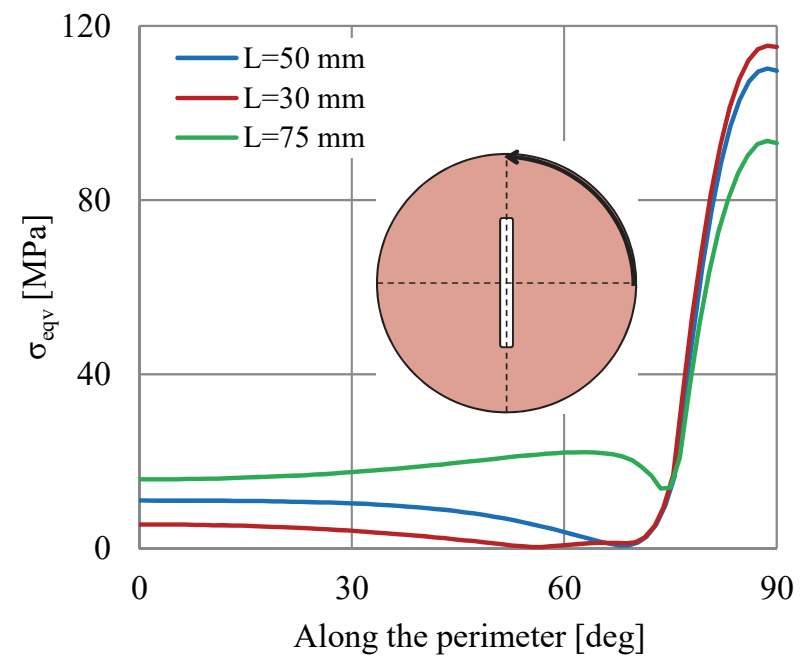

(b)

Figure 14: The variation of the maximum principal stress along one quarter of the notch with a detailed view around its corner (a); and the variation of the equivalent stress around one quarter of the perimeter of the disc (b).

applied load, is plotted in Fig.15, versus the width of a notch machined in a disc of radius 50 mm with a notch of length equal to $50 \mathrm{~mm}$, i.e., equal to the radius of the disc. Two strategic points are considered, i.e., point $\mathrm{M}$ on the longitudinal axis of symmetry of the notch and point $\mathrm{C}$, the mid-point of the rounded corner of the notch. It is definitely seen that criticality is achieved at point $\mathrm{M}$ where the stress concentration is considerably exceeding that at point $\mathrm{C}$, and therefore crack is indeed expected to start propagating along the symmetry axis of the configuration (in full accordance with a series of experimental results [13]).

It is seen from Fig.15 that even for widths of the notch smaller than $0.5 \mathrm{~mm}$ (a value that is well below the limits of any practical method used to machine the notch) the maximum equivalent stress developed is about 4 times higher compared to the amplitude of the externally applied parabolic stress distribution, and by no means it could be considered as tending to infinite values.

Recapitulating, it can be definitely stated that the stress field developed in the disc is strongly influenced by the geometric characteristics of the notch especially in the vicinity of its crowns. Obviously, ignoring the exact geometric shape of the notch may lead to erroneous results concerning the actual severity of the stress field in this critical region and therefore it is quite possible that misleading results about the actual fracture toughness of the disc's material will be obtained. 


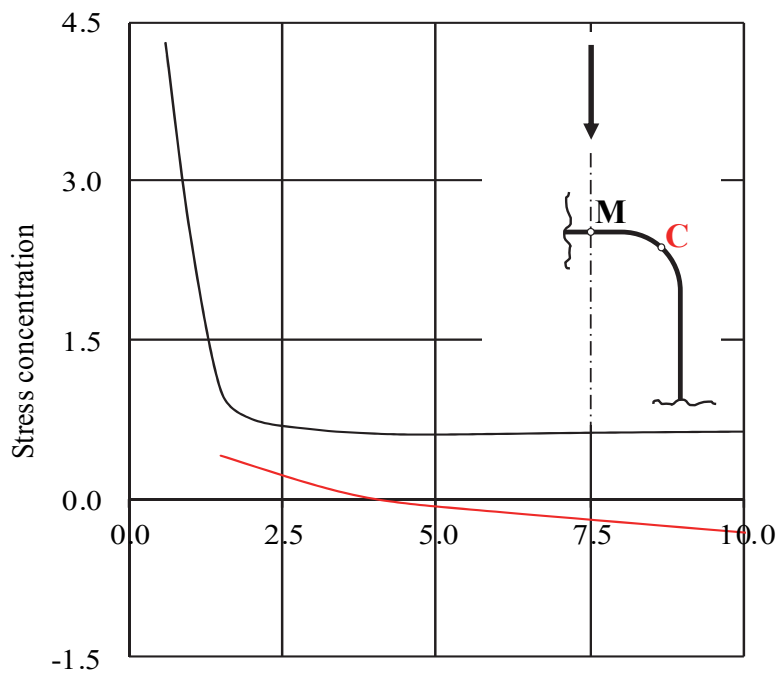

Width of the notch, w [mm]

Figure 15: The stress concentration (i.e., the ratio of the equivalent stress developed over the amplitude of the externally applied load) versus the width of a notch in a disc of radius $50 \mathrm{~mm}$ with a notch of length equal to $50 \mathrm{~mm}$.

\section{ACKNOWLEDGEMENTS}

his research is co-financed by Greece and the European Union (European Social Fund - ESF) through the Operational Programme «Human Resources Development, Education and Lifelong Learning 2014-2020» in the context of the project "Critical assessment and revision of the standard regarding the determination of the Mode I fracture toughness, $\mathrm{K}_{\mathrm{IC}}$, of brittle building materials using a combination of innovative experimental techniques, numerical simulations and analytical methods" (MIS 5049181).

\section{REFERENCES}

[1] ASTM (2014). E399-12e3 Standard test method for linear-elastic plane-strain fracture toughness $\mathrm{K}_{\mathrm{IC}}$ of metallic materials. ASTM volume 03.01: Metals-Mechanical Testing; Elevated and Low Temperature Tests; Metallography.

[2] ISRM (1995). Suggested methods for determining Mode-I fracture toughness using CCNBD specimens, Int. J. Rock Mech. Min., 32(1), pp. 57-64.

[3] Kourkoulis, S.K. and Markidis, Ch.F. (2014). Fracture toughness determined by the centrally cracked Brazilian disc test: Some critical issues in the light of an alternative analytic solution, ASTM Materials Performance \& Characterization, 3(3), pp. 45-86. DOI: 10.1520/MPC20130056.

[4] Markides, Ch.F. and Kourkoulis, S.K. (2016). "Mathematical" cracks versus artificial slits: Implications in the determination of fracture toughness, Rock Mech. Rock Eng., 49(3), pp. 707-729. DOI: 10.1007/s00603-015-0794-y.

[5] Fowell, R.J., Xu, C. and Dowd, P.A. (2006). An update on the fracture toughness testing methods related to cracked CCNBD specimen, Pure Appl. Geophys., 163, pp. 1046-1057. DOI: 10.1007/s00024-006-0057-7.

[6] Kaklis, K., Mavrigiannakis, S., Saltas, V., Vallianatos, F. and Agioutantis, Z. (2017). Using acoustic emissions to enhance fracture toughness calculations for CCNBD marble specimens. Frattura ed Integrità Strutturale, 11(40), pp. 1-17. DOI: 10.3221/IGF-ESIS.40.01.

[7] Kourkoulis, S.K., Markides, Ch.F. and Chatzistergos, P.E. (2013). The standardized Brazilian disc test as a contact problem, Int. J. Rock Mech. Min., 57, pp. 132-141. DOI: 10.1016/j.ijrmms.2012.07.016.

[8] Markides, Ch.F. and Kourkoulis, S.K. (2013). Naturally accepted boundary conditions for the Brazilian disc test and the corresponding stress field, Rock Mech. Rock Eng., 46(5), pp. 959-980. DOI: 10.1007/s00603-012-0351-x.

[9] Muskhelishvili, N.I. (1963). Some Basic Problems of the Mathematical Theory of Elasticity, Noordhoff, Groningen, The Netherlands.

[10] Markides, Ch.F. and Kourkoulis, S.K. (2015). The displacement field in a finite circular disc with a central rectangular slit, Procedia Engineering, 109, pp. 257-267. DOI: 10.1016/j.proeng.2015.06.231. 
[11] Savin, G.N. (1970). Stress Distribution Around Holes, NASA, TT F-607, Washington DC.

[12] Kourkoulis, S.K., Markides, Ch.F. and Pasiou, E.D. (2015). A combined analytic and experimental study of the displacement field in a circular ring, Meccanica, 50(2), pp. 493-515. DOI: 10.1007/s11012-013-9846-0.

[13] Kourkoulis, S.K., Pasiou, E.D., Loukidis, A., Stavrakas, I. and Triantis, D. (2021). The determination of mode-I Fracture Toughness (by means of the Brazilian-Disc configuration) in the light of experimental evidence provided by novel sensing techniques. SyMBoL final conference, Online, 2-3 September. 